\title{
Rigid Point Feature Registration for MRI Brain Images based on Fuzzy Transform
}

\author{
Nemir Ahmed Al-Azzawi \\ Baghdad University \\ Electrical Engineering Department \\ Al-Jadriah, P.O.Box 47187 Baghdad, Iraq.
}

\begin{abstract}
Radiological images are gradually more being used in healthcare and medical research. There is, accordingly, widespread interest in accurately relating information in the different images for diagnosis, treatment and basic science. In this paper we formulate feature registration problems by using F-transform (Fuzzy-Transform) to extract the salient edges and extracted control points (CP) of medical Images. The MRI brain Images were first decomposed using F-transform, then Edge and CP were extracted from F-transform error function, and some proposed rules. After edge and CP extraction, mutual information (MI) was adopted for the registration of feature points, and translation parameters are calculated by using particle swarm optimization (PSO). We implement experiments to evaluate the performance of the FTransform and MI similarity measures for 2-D/3-D rigid point feature registration. The experimental results showed that the proposed method produces totally accurate performance for rigid point feature registration for MRI brain images.
\end{abstract}

\section{Keywords}

Feature-based registration; mutual information; FuzzyTransform (F-Transform); particle swarm optimization (PSO).

\section{INTRODUCTION}

Medical image registration is a fundamental task in medical image analysis. In the particular case of the brain, there are a number of significant applications including comparing shape and function between individuals or groups, developing probabilistic models and atlases, measuring change within an individual and determining location with respect to a pre acquired image during stereotactic surgery. Brain imaging is particularly essential as well for the knowledge of the functional and/or pathological processes, as for the improvement of adapted strategies of treatment. There are, therefore, possible benefits in improving the way in which these images are compared and combined (image fusion) [1]. Current clinical practice normally involves printing the images onto radiographic film and viewing them on a light box. Computerized approaches offer potential benefits, particularly by accurately aligning the information in the different images, and providing tools for visualizing the combined images. Thus, these last years, many researchers predictable the need to develop treatment tools in order to assist the expert in his diagnosis choice. Generally, it is a clinical aided system dedicated to make the assessment. For example, in neurosurgery it is currently helpful to identify tumors with magnetic resonance images (MRI). The goal of image registration is to find an optimal geometric transformation between corresponding image data [2], where the criteria for optimality depends on specific application. A critical stage in this process is the alignment or registration of the images, which is the topic of this paper.

Previous work on medical image registration can be characterized based on the used image information into intensity-based methods and feature-based [3]. The first class utilizes image intensity to estimate the parameters of a transformation between two images using an approach involving all pixels of the image. In contrast, the second class does not work directly with image intensity values and rely on establishing feature correspondence between the two images. The feature-based matching algorithm may be performed by iterative closest point (ICP) algorithms [4] or by optimizing deformable models [5]. This methods, firstly uses feature matching techniques to determine corresponding feature pairs from the two images, and then compute the geometric transformation relating them

The accuracy of registration algorithm is consequently affected by the segmentation and feature extraction algorithms [3]. Researching and exploring more accurate and faster registration algorithm is a very important domain. The main advantage of feature-based method, where a matching algorithm is sought between corresponding objects within the images, is approximately invariance for the intensity characteristics of the pixels. This method is sensitive to the error of feature extraction and matching [6]. One general approach for extracting information from the image is to reduce the quantity of data by making abstractions such as edges. For many years, edge detection has been proved a very valuable tool for automating the extraction of information at a low level. The aim of edge detection is to extract features, as meaningful as possible depending on the physical illumination properties of an image. Edge detection is the most common method for feature extraction. The problem of edge detection is one of the most attractive problems for the image processing community due to various important applications. Canny edge detection is commonly used to generate features for image registration to handle the accurate feature extraction [7-8]. The F-transform [9-10] is an efficient intelligent method to handle uncertain information. It represents those natural phenomena which we observe in our real lives. Daňková and Valášek [11] shows that the F-transform technique is a promising and efficient method for feature and edge extraction.

Mutual information (MI)-based image registration has been found to be quite effective in many medical imaging 
applications. It is ordinary to ask whether mutual information can play a comparable role in feature-based matching as well. Given that both MR and CT are informative of the same underlying anatomy, there will be mutual information between the MR image and the CT image. Rangarajan [8] demonstrates that mutual information can be utilized to parameterize and solve the correspondence problem in feature-based registration. The mutual information (similarity measured) will also be a function of the spatial mapping and can be maximized using an appropriate optimization algorithm. The choice of optimization method is very much linked to the characteristics of the accuracy of registration. Most optimization methods rely on some user-defined parameters. Incorrect settings of these parameters may lead to an excessive computation time, low precision, or even divergence of the solution. Powell's direction set method [12] and downhill simplex method [13] are used when local distortion is not present. Particle swarm optimization (PSO) has been proposed as global optimization technique [14-15], which is a stochastic, population-based evolutionary computer algorithm.

In this paper, MRI brain Images registration has been presented based on F-transform, MI and PSO. The medical images were first decomposed using the F-transform, then edge and $\mathrm{CP}$ was extracted from bandpass directional subband of F-transform coefficients and some adjacent rules. After edge and CP extraction, mutual information was adopted for the registration of feature points and transformation parameters are calculated by using particle swarm optimization (PSO). There are three main steps carried out for proposed feature-based image registration, edge detection using F-transform, optimization the MI based on particle swarm optimization and transformation parameters estimation. The experimental results demonstrate the robustness, efficiency and accuracy of the algorithm.

\section{F-Transform}

The original motivation for the F-transform (an abbreviated name for the fuzzy transform) came from fuzzy modeling Perfilieva [9-10]. The intention was to show that, similarly to conventional transforms (Fourier and wavelet).

Let $u$ be represented by the discrete function $u: P \rightarrow \mathbb{R}$ of two Variables, where $P=\{(i, j) \mid i=1, \ldots, N, j=$ $1, \ldots, M\}$ is an $N \times M$ array of pixels, and $\mathbb{R}$ is the set of reals. If $(i, j) \in P$ is a pixel, then $u(i, j)$ represents its intensity range. Moreover, let fuzzy sets $A_{k} \times B_{l}, k=1, \ldots, n, l=1, \ldots, m$, where $0<n \leq N, 0<m \leq M$ establish a fuzzy partition of $[1$, $N] \times[1, M]$. The F-transform of $u$ corresponds $u$ to the matrix $F[u]_{n m}$ of F-transform components:

$F[u]_{n m}=\left(\begin{array}{ccc}F[u]_{11} & \ldots & F[u]_{1 m} \\ \vdots & \vdots & \vdots \\ F[u]_{n 1} & \ldots & F[u]_{n m}\end{array}\right)$

Each component $F[u]_{k l}$ is a local mean value of $u$ over a support set of the respective fuzzy set $A_{k} \times B_{l}$. The membership functions of the respective fuzzy sets in a fuzzy partition are called basic functions. The (direct) F-transform of $u$ (with respect to the chosen partition) is an image of the mapping $F[u]:\left\{A_{1}, \ldots, A_{n}\right\} \times\left\{B_{1}, \ldots, B_{m}\right\} \rightarrow \mathbb{R}$ defined by:

$$
F[u]\left(A_{k} \times B_{l}\right)=\frac{\sum_{i=1}^{N} \sum_{j=1}^{M} u(i, j) A_{k}(i) B_{l}(j)}{\sum_{i=1}^{N} \sum_{j=1}^{M} A_{k}(i) B_{l}(j)}
$$

where $\mathrm{k}=1, \ldots, n, 1=1, \ldots, m$. The value $F[u]\left(A_{k} \times B_{l}\right)$ is called an F-transform component of $u$ and is denoted by
$F[u]_{k l}$. We now introduce two extreme fuzzy partitions of $[1$, $N]$ that will be used in the following.

\section{Largest partition:}

The largest partition of $[1, N] \times[1, M]$ contains only one fuzzy set, $A_{1} \times B_{1}$, such that for all $(x, y) \in[1, N] \times[1, M],\left(A_{1} \times\right.$ $\left.B_{1}\right)(x, y)=1$. The respective F-transform component $F[u]_{11}$ and the respective inverse F-transform $u_{11}$.

\section{Finest partition:}

The finest partition of $[1, N] \times[1, M]$ is established by $N \times M$ fuzzy sets $A_{k} \times B_{l}$, such that for all $k=1, \ldots, N$, and $l=1, \ldots, M$. The respective F-transform components $F[u]_{k l}, k=1, \ldots, N$, $l=1, \ldots, M$, and the respective inverse F-transform $u_{N M}$. The inverse F-transform of $u$ is a function on $P$, which is represented by the following inversion formula:

$$
u_{n m}(i, j)=\sum_{i=1}^{n} \sum_{j=1}^{m} F[u]_{k l} A_{k}(i) B_{l}(j)
$$

where $i=1, \ldots, N, j=1, \ldots, M$. It can be shown that the inverse F-transform, $u_{n m}$ approximates the original function $u$ on the domain $P$. The proof can be found in $[9,16-17]$. The Ftransform technique, leading to one-level or higher-level decomposition of an image; here we explain the technical details of these decompositions. The one level decomposition is as the following representation of $u$ on $P$ :

$$
\begin{gathered}
u(x, y)=u_{n m}(x, y)+e(x, y) \\
e(x, y)=u(x, y)-u_{n m}(x, y), \forall(x, y) \in P
\end{gathered}
$$

where $0<n \leq N, 0<m \leq M$ and $u_{n m}$ is the inverse F-transform of $u$ and $e(x, y)$ is error function the respective residuum. If fuzzy sets $A_{1}, \ldots, A_{n}$ establish a fuzzy partition of $[1, N]$ and $B_{1}, \ldots, B_{m}$ do the same for $[1, M]$ then the Cartesian product $\left\{A_{1}, \ldots, A_{n}\right\} \times\left\{B_{1}, \ldots, B_{m}\right\}$ of these fuzzy partitions is the set of all fuzzy sets $A_{k} \times B_{l}, k=1, \ldots, n, l=1, \ldots, m$ and $h=\frac{N-1}{n-1}$ a distance between nodes $x_{1}, \ldots, x_{n} \in[1, N]$, where $x_{1}=1$, $x_{k}=x_{1}+(k-1) h, k=1, \ldots, n$. The membership function $A_{k} \times B_{l}:[1, N] \times[1, M] \rightarrow[0,1]$ is equal to the product $A_{k}$. $B_{l}$ of the respective membership functions. The difference between an original function and its inverse F-transform works as a high-pass filter of the former. Therefore, the mentioned above difference can be used for the edge detection problem [11].

\section{The Proposed Registration Algorithm}

Specified two images, $I_{R}$ (defined as a reference image) and $I_{U}$ (defined as a unaligned image) to match the reference image, the goal of image registration is to fix the unaligned image into the coordinate system of the reference image and to make corresponding coordinate points in the two images fit the same geographical location. In this section, we present the registration algorithm. There are three main steps carried out for registration

\subsection{Edge Detection using F-transform}

During arrange to extract two sets of feature points, $C P 1_{i}(i=1$, $2, \ldots N 1)$ and $C P 2_{i}(i=1,2, \ldots N 2)$ from the reference and the unaligned images respectively, a F-transform based feature points extraction method is employed. The method can be summarized by the following algorithm:

1. Calculate $F[u]$ - the direct F-transform of image $u: P \rightarrow$ $\mathbb{R}$, by (2), for both unaligned and reference image.

2. Calculate $u_{n m}$ - the inverse F-transform using the components $F[u]$ by (3). 
3. Calculate the error function $e(x)=\left|u(x)-u_{n m}(x)\right|$ for all $x \in P$.

4. Rescale and round the values of $e$ from $\left[0, \max _{x \in P} e(x)\right]$ the integers in $[0,255]$, which results in the new image $e_{r}$ . Output: Image $e_{r}$

5. Control points $C P$ is found by applying a threshold procedure to both error function $e(x)$ for reference and unaligned image respectively. Using threshold as in the following rule:

$$
T h_{j}=c\left(\sigma_{j}+\mu_{j}\right)
$$

where, $c$ is a constant defined by the user and $\sigma_{j}$ and $\mu_{j}$ are the standard deviation and mean of the error function image. A low standard deviation indicates to be very close to the same value (the mean), while high standard deviation indicates that the data are spread out over a large range of values.

6. The locations of the obtained threshold error function $C P 1_{i}(i=1,2, \ldots, N 1)$ and $C P 2_{i}(i=1,2, \ldots, N 2)$ are taken as the extracted feature points. where $C P 1_{i}, C P 2_{\mathrm{i}}$ are the coordinates and $N 1, N 2$ are the number of feature points.

\subsection{Optimization the MI based on PSO}

Mutual information (MI) is the most popular image similarity measures for registration of multimodality images [18-21]. The implementation of MI are discussed particularly in [8]. Once the feature points of two images have been extracted, mutual information is adopted as a similarity measure to be optimized. For two images $A$ and $B$, mutual information $I$ can be defined as :

$$
I(A, B)=H(A)+H(B)-H(A, B)
$$

where $H(A)$ is the Shannon entropy of image $A$, Computed on the probability distribution of the grey values. $H(A, B)$ is joint entropy [18]. This form contains the term $-H(A, B)$, which means that maximizing mutual information is related to minimizing joint entropy. Let $X_{i}=\left\{X_{i}\right.$ i $\left.=1,2, \ldots, N_{l}\right\}$ be points of F-transform error function reference image and $Y_{j}=\{$ $\left.Y_{j}, j=1,2, \ldots, N_{2}\right\}$ be points of F-transform error function unaligned image. The mutual information between the pointsets is a function of the joint probability as follows:

$$
M I(X, Y)=\sum_{i=1}^{N 1} \sum_{j=1}^{N 2} P_{i j} \log \frac{P_{i j}}{\sum_{k=1}^{N 1} P_{k j} \sum_{l=1}^{N 2} P_{i l}}
$$

where the joint probability $P_{i j}$ is the association probability between indices.

The particle swarm optimization (PSO) algorithm is used to find the maximum $M I(X, Y)$. The PSO algorithm uses an inhabitant of particles which travel in a multidimensional space that represents the space of solutions for the problem[15]. In PSO, each answer of problem, called particle, flies in the D-dimensional space with the velocity dynamically adjusted according to the individual information and population information. It has been proposed as global optimization technique [14-15], which is a stochastic, population-based evolutionary computer algorithm. The Basic PSO algorithm consists of the velocity:

$$
\begin{aligned}
& v_{i}(k+1)=v_{i}(k)+\gamma_{1 i}\left(p_{i}-x_{i}(k)\right)+\gamma_{2 i}\left(p_{g}-x_{i}(k)\right) \\
& \text { Position: } x_{i}(k+1)=x_{i}(k)+v_{i}(k+1)
\end{aligned}
$$

where, $(i)$ is particle index, $(k)$ is discrete time index, $(v)$ is velocity of $i^{\text {th }}$ particle, $(x)$ is position of $i^{\text {th }}$ particle, $(p)$ is best position found by $i^{\text {th }}$ particle (personal best), $\left(p_{g}\right)$ is best position found by swarm (global best, best of personal bests) and $\left(\gamma_{1 i}, \gamma_{2 i}\right)$ are random numbers on the interval $[0,1]$ applied to $i^{\text {th }}$ particle. The PSO can be easily extended. We assume a set of $\mathrm{m}$ particles in $\mathrm{D}$-dimensional searching space, in which the first particle stands for a D-dimensional vector $\vec{x}_{i}=\left(x_{i 1}, x_{i 2}, \ldots, x_{i D}\right), i=1, \ldots, m$ it is the position of $\vec{x}_{i}$. In other terms, every position is a prospective resolution. We can get a corresponding value if $\vec{x}_{i}$ is set to the target function. Set the present optimal position of the first particle is $\vec{p}_{i}=$ $\left(p_{i 1}, p_{i 2}, \ldots, p_{i D}\right), i=1, \ldots, m$. The present optimal position of the swarm is $\vec{p}_{g}=\left(p_{g 1}, p_{g 2}, \ldots, p_{g D}\right), i=1, \ldots, m$. Operating the particles with the formulas:

$$
\begin{gathered}
v_{i}(k+1)=\emptyset(k) v_{i}(k)+\alpha_{1}\left[\gamma_{1 i}\left(p_{i}-x_{i}(k)\right)\right]+ \\
\alpha_{2}\left[\gamma_{2 i}\left(p_{g}-x_{i}(k)\right)\right], i=1, \ldots, m
\end{gathered}
$$

where, $(\varnothing)$ is Inertia function and $\left(\alpha_{1,2}\right)$ is nonnegative acceleration constants. $v_{i} \in\left[-v_{\max }, v_{\min }\right), v_{\max }$ is a constant value and it is set by the user. The ending condition of the iteration is essentially decided by the largest iteration or the threshold of the optimal position of the particles. We take the MI, which is depending on the parameters as the value of the target function.

3.3 Transformation Parameters Estimation Image geometrical deformation has many different ways of description [6, 22-23]. Combination of rotation, scaling and translation is the most common one and it has five parameters: $\left\{t_{x}, t_{y}\right\}$ translation, rotation $\{\theta\}$, and scale $\left\{s_{x}, s_{y}\right\}$. This type of transformations is considered. Given the two sets of corresponding feature point coordinates optimum $C P$, the estimation of the transformation parameters, required to transform the unaligned image into its original size, direction, and position.

\section{Numerical Experiments}

The proposed methods has been implemented and tested on medical images provided by Hospital Universiti Sains Malaysia (HUSM). To validate and test the registration systems, numerous experiments were conducted with images of different dimensions and pixel resolutions. Thirty groups with different subjects of human brain images were selected (only six subjects see Table I). Registration systems were implemented in MATLAB software package. Clinical MRI, T1 image volumes were acquired with an echo time (TE) of $11 \mathrm{~ms}$ and a repetition time (TR) of $420 \mathrm{~ms}$, using a GE Signa Horizon 1 Tesla scanner. MRI image is used as reference and subject images as unaligned. The images have the resolution $(x=512, y=512$ and $z=20-24)$ with 256 -level grayscale and voxel size $(x=1.25, y=1.25$ and $z=5 \mathrm{~mm})$. Subject images were synthetically generated by adjusting the transformation parameters relative to the reference image. $\left\{t_{x}, t_{y}, \theta, s_{x}, s_{y}\right\}$ are translations, rotates and scales in $x, y$ directions. Table I, are indicates the spatial relations between the reference image and subject images. In F-transform, the input arguments $n ; m$ are determine the thickness of the edge. Hence, the bigger are $n ; m$ the thinner are edges. It follows that the edges of all objects in focus can be determined from the change of intensities of the pixels of the closest neighbor-hoods, i.e. setting $n ; m$ such that each $A_{i} ; B_{j}$ covers min. 4 pixels (i.e. $h>2$ pixels). The experiment was carried out 120 pixels and $c=1.8$.

For the both canny edge extraction and F-transform edge extraction. The initial transformation parameters 
$\left\{t_{x}, t_{y}, \theta, s_{x}, s_{y}\right\}$ were bounded in the following way $\{-20<$ $\left.t_{x}, t_{y}<20 ;-15^{\circ}<\theta<15^{\circ} ; 0.5<s_{x}<2 ; 0.5<s_{y}<2\right\}$ An experimental result was compared with method [8] based on canny edge method. Registration result for canny edge extraction and F-transform edge extraction is shown in Fig. 2. The parameters of affine transformation matrix output from two registration systems were listed in Table II.

In this test series, two registration systems provided perfect registrations for single subject with image sources from same modality. However, for canny edge extraction and Ftransform edge extraction, good initial search location needs to be provided within the normal range of misregistration. Fig. 1 , it shows the target registration error (TRE) in (mm) in all images tests. It can be easily shown in Fig. 2, that the registration results based on F-transform is ideal accurate. So the method proposed in the paper is applicable in the medical image registration system.

\section{Conclusions}

In this paper, we have introduced a MRI brain Images registration, which are employing F-transform and mutual information. As a result, the registration performance was perfect. The speed of finding for the optimum value is also improved after using PSO. According to the experiments, we can conclude that the method proposed is maintaining excellent performance; however, experiments show that our method works well for MRI brain Images registration. In a comparison with standard methods we have demonstrated that the edge detection algorithm based on F-transform performs satisfactorily well.

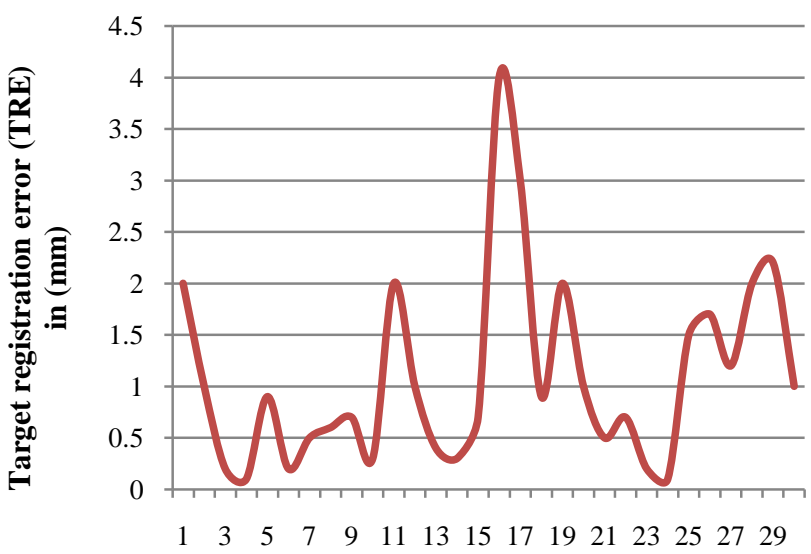

Fig 1: The target registration error (TRE) in $(\mathrm{mm})$ in all images tests.
TABLE 1. Indicates the spatial relations between the reference image and subject images.

\begin{tabular}{|c|c|c|c|c|c|}
\hline \multirow{2}{*}{ Subjects } & \multicolumn{5}{|c|}{ Parameters } \\
\cline { 2 - 6 } & $\mathbf{T}_{\mathbf{x}}$ & $\mathbf{T}_{\mathbf{y}}$ & $\boldsymbol{\theta}$ & $\mathbf{s}_{\mathbf{x}}$ & $\mathbf{s}_{\mathbf{y}}$ \\
\hline Subject 1 & 20 & & & & \\
\hline Subject 2 & 15 & 20 & & & \\
\hline Subject 3 & 15 & 20 & 5 & & \\
\hline Subject 4 & 20 & 20 & 10 & & \\
\hline Subject 5 & 15 & 20 & 15 & 1.2 & \\
\hline Subject 6 & 15 & 20 & 5 & 1.2 & 1.2 \\
\hline
\end{tabular}

TABLE 2. The outputs from proposed F-Transform feature-based image registration algorithm and canny method.

\begin{tabular}{|l|c|c|c|c|c|}
\hline \multirow{2}{*}{ Subjects } & \multicolumn{5}{|c|}{ F-transform method } \\
\cline { 2 - 6 } & $\boldsymbol{T}_{\boldsymbol{x}}$ & $\boldsymbol{T}_{\boldsymbol{y}}$ & $\boldsymbol{\theta}$ & $\boldsymbol{s}_{\boldsymbol{x}}$ & $\boldsymbol{s}_{\boldsymbol{y}}$ \\
\hline Subject 1 & 20 & & & & \\
\hline Subject 2 & 15 & 20 & & & \\
\hline Subject 3 & 14.99 & 20 & 5 & & \\
\hline Subject 4 & 19.89 & 19.99 & 10 & & \\
\hline Subject 5 & 14.99 & 20 & 15 & 1.2 & \\
\hline Subject 6 & 15 & 19.9 & 5 & 1.2 & 1.2 \\
\hline & & \multicolumn{5}{|c|}{ Canny method } & \\
\hline Subject 1 & 19.12 & & & & \\
\hline Subject 2 & 13.23 & 18.88 & & & \\
\hline Subject 3 & 13.22 & 19.21 & 4.62 & & \\
\hline Subject 4 & 19.21 & 18.99 & 9.18 & & \\
\hline Subject 5 & 13.66 & 19.23 & 14.83 & 1.10 & \\
\hline Subject 6 & 14.22 & 19.33 & 4.96 & 1.18 & 0.92 \\
\hline
\end{tabular}




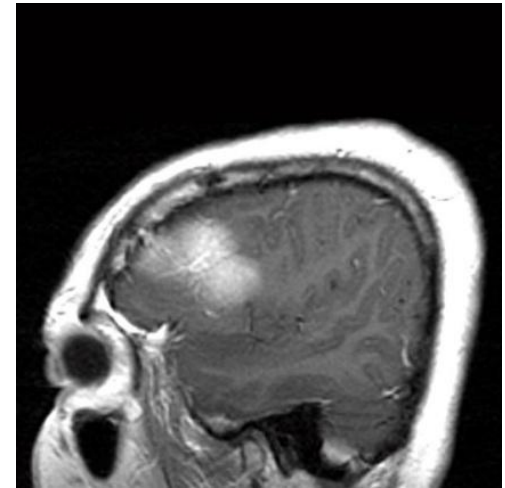

(a)

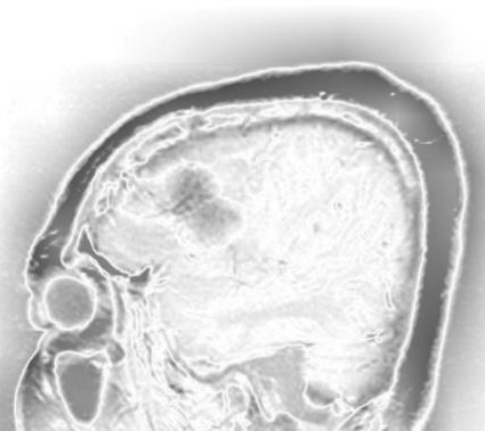

(d)

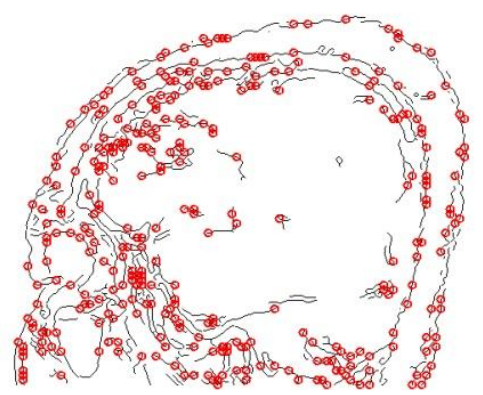

(g)

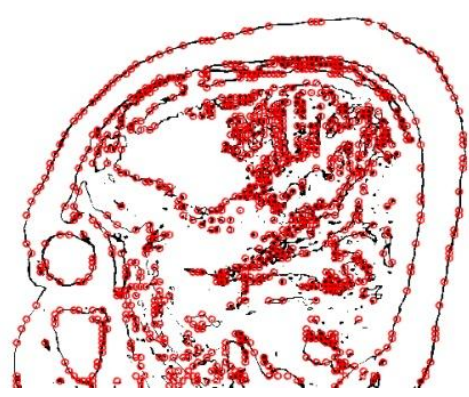

(g)

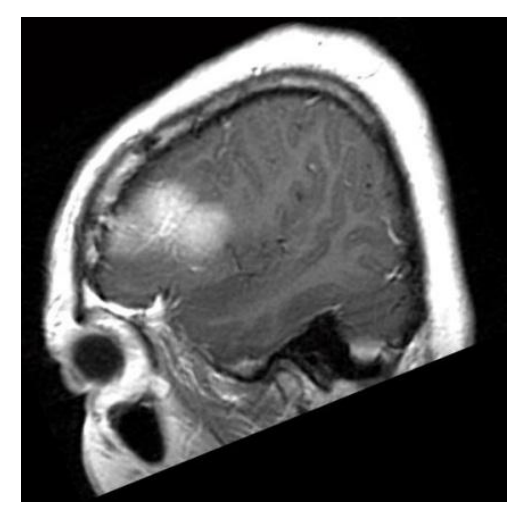

(b)

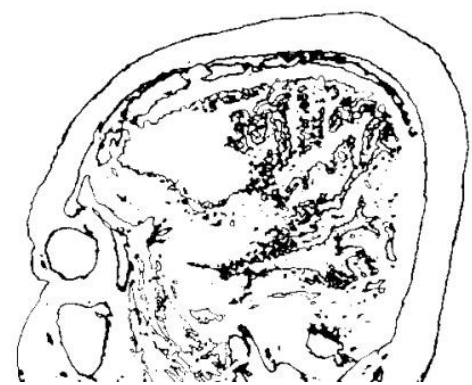

(e)

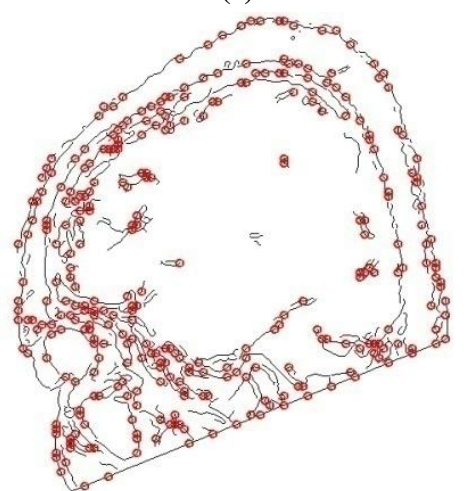

(h)

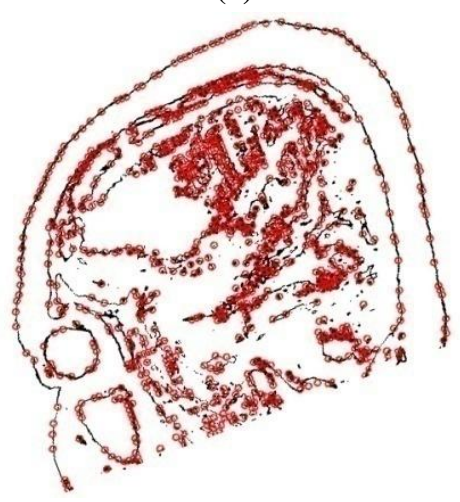

(k)

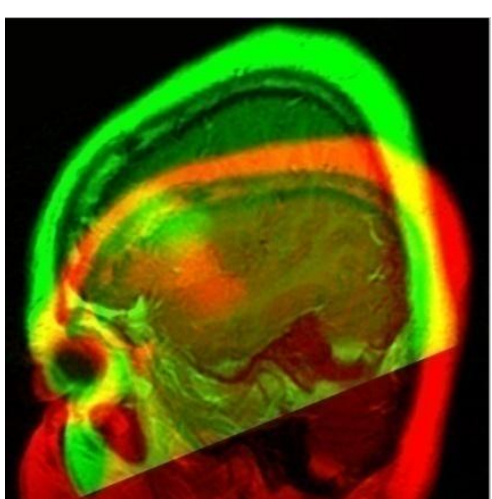

(c)

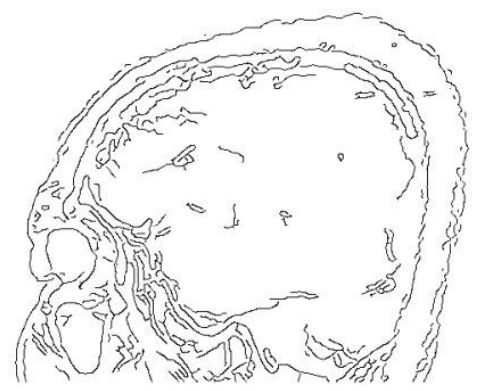

(f)

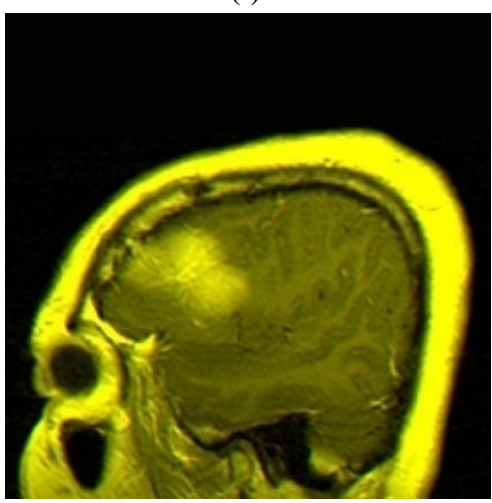

(i)

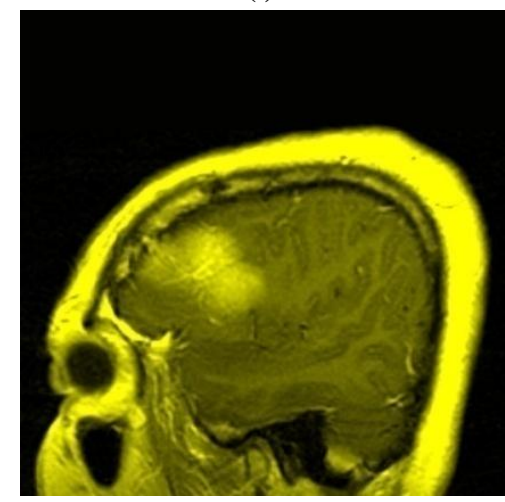

(l)

Fig 2: Registration result for canny edge extraction and F-transform edge extraction. (a) Reference MRI, (b) Unaligned, (c) Fused MI=0.482, (d) F-transform algorithm, $e_{r}$ with 100 pixels covered, (e) F-transform algorithm, $e_{r}$ with 100 pixels covered and threshold [11], (f) Canny algorithm, (g) Canny Extracted points, (h) Canny Extracted points, (i) Canny-Registered MI=1.121, (g) F-transform Extracted points, (k) F-transform Extracted points and (l) F-transform Registered MI=1.932. 


\section{References}

[1] N. Al-Azzawi, et al., "Medical image fusion scheme using complex contourlet transform based on PCA," in Annual International Conference of the IEEE Engineering in Medicine and Biology Society, EMBC 2009, Hilton Minneapolis, Minnesota, USA, 2009, pp. 5813-5816.

[2] J. V. Hajnal, et al., Medical image registration. Boca Raton: CRC Press, 2001.

[3] A. Goshtasby, 2-D and 3-D image registration for medical, remote sensing, and industrial applications. Hoboken, NJ: J. Wiley \& Sons, 2005.

[4] S. Rusinkiewicz and M. Levoy, "Efficient variants of the ICP algorithm," in Proceedings Third International Conference on 3-D Digital Imaging and Modeling, Quebec City, 2001, pp. 145-152.

[5] E. Meijering, et al., "Tracking in molecular bioimaging," IEEE Signal Processing Magazine, vol. 23, pp. 46-53, 2006.

[6] B. Zitova and J. Flusser, "Image registration methods: A survey," Image and Vision Computing, vol. 21, pp. 9771000, 2003.

[7] J. Canny, "A computational approach to edge detection," IEEE Transactions on Pattern Analysis and Machine Intelligence, vol., PAMI-8, pp. 679-698, Nov. 1986.

[8] A. Rangarajan, et al., "Rigid point feature registration using mutual information," Medical Image Analysis, vol. 3, pp. 425-440, 1999.

[9] I. Perfilieva, "Fuzzy transforms: Theory and applications," Fuzzy Sets and Systems, vol. 157, pp. 9931023, 2006.

[10] I. Perfilieva, et al., "Image fusion on the basis of fuzzy transforms," in The 8th International FLINS Conference on Computational Intelligence in Decision and Control, Madrid, Spain, 2008, pp. 471-476.

[11] M. Dankova, et al., "Edge detection using F-transform," in Intelligent Systems Design and Applications (ISDA), 2011 11th International Conference on, 2011, pp. 672677.

[12] F. Yang and H.-L. Zhang, "Multiresolution 3D image registration using hybrid ant colony algorithm and powell's method," Journal of Electronics and Information Technology, vol. 29, pp. 622-625, 2007.
[13] A. V. Tuzikov, et al., "Evaluation of the symmetry plane in 3D MR brain images," Pattern Recognition Letters, vol. 24, pp. 2219-2233, 2003.

[14] B. Birge, "PSOt - a particle swarm optimization toolbox for use with Matlab," in Proceedings of the 2003 IEEE Swarm Intelligence Symposium, 2003. SIS '03, Indianapolis (IN), USA, 2003, pp. 182-186.

[15] J. Kennedy and R. C. Eberhart, "Particle swarm optimization," in Proceedings of IEEE International Conference on Neural Networks, Piscataway, NJ, 1995, pp. 1942-1948.

[16] I. Perfilieva, "Fuzzy transforms: A challenge to conventional transforms," in Advances in Images and Electron Physics. vol. 147, ed San Diego: Elsevier Academic Press, 2007, pp. 137-196.

[17] I. Perfilieva and R. Valášek, "Fuzzy Transforms in Removing Noise

Computational Intelligence, Theory and Applications." vol. 33, B. Reusch, Ed., ed: Springer Berlin / Heidelberg, 2005, pp. 221-230.

[18] J. P. W. Pluim, et al., "Mutual-information-based registration of medical images: a survey," IEEE Transactions on Medical Imaging, vol. 22, pp. 986-1004, 2003.

[19] A. Gholipour, et al., "Brain functional localization: A survey of image registration techniques," IEEE Transactions on Medical Imaging, vol. 26, pp. 427-451, 2007.

[20] F. Maes, et al., "Multimodality image registration by maximization of mutual information," IEEE Transactions on Medical Imaging, vol. 16, pp. 187-198, 1997.

[21] N. Al-Azzawi and W. A. K. W. Abdullah, "MRI Monomodal Feature-Based Registration Based on the Efficiency of Multiresolution Representation and Mutual Information," American Journal of Biomedical Engineering, vol. 2, pp. 98-104, 2012.

[22] L. G. Brown, "A survey of image registration techniques," ACM Computing Surveys, vol. 24, pp. 325 376, 1992.

[23] A. E. R. Arce-Santana and A. Alba, "Image registration using Markov random coefficient and geometric transformation fields " Pattern Recognition vol. 42, pp. 1660-1671, 2009 